Bio - grafía. Escritos sobre la Biología y su Enseñanza. ISSN 2027-1034

Edición Extraordinaria. p.p. 1221 - 1229

Memorias del IX Encuentro Nacional de Experiencias en Enseñanza de la Biología y la Educación Ambiental. IV Congreso Nacional de Investigación en Enseñanza de la Biología.

\title{
EL TRABAJO COOPERATIVO EN EL FORTALECIMIENTO DE COMPETENCIAS CIENTÍFICAS PARA LA COMPRENSIÓN DE LAS CIENCIAS NATURALES
}

\author{
Yolanda Emilce Olaya Conde ${ }^{1}$ \\ Carlos Humberto Barreto Tovar ${ }^{2}$
}

\section{RESUMEN}

Esta investigación muestra la experiencia del trabajo realizado en el aula de clase desde el campo de Ciencia y Tecnología con estudiantes de grado Séptimo en el Colegio Pablo de Tarso I.E.D. El trabajo de aula indagó sobre el desarrollar competencias científicas y tecnológicas desde el enfoque de la Enseñanza para la Comprensión (EpC) a partir de la metodología del aprendizaje cooperativo. Describe la ruta de trabajo establecida, las ventajas y desventajas de la metodología empleada, el proceso de aprendizaje realizado, las competencias científicas adquiridas, el uso de herramientas tecnológicas y los mecanismos de interacción para la formación de ciudadanos.

A partir de la revisión conceptual y su contraste con los ejercicios realizados en clase, se pudo establecer las características de la práctica docente con los estudiantes, generando procesos de mejora y de aceptación de la metodología de trabajo cooperativo que favorecen y optimizan el aprendizaje en ciencias naturales mediante la comprensión de los contenidos planteados y la socialización asertiva.

PALABRAS CLAVES: Enseñanza para la Comprensión - Aprendizaje Cooperativo - Competencias Científicas - Formación de Ciudadanos - Uso de las TIC.

\section{ABSTRACT}

This research shows the experience of the work done in the classroom from the field of Science and Technology with students of seventh grade in the School Pablo de Tarso I.E.D. The classroom work investigated the development of

\footnotetext{
${ }^{1}$ Docente de la Secretaría de Educación de Bogotá. Colegio Pablo de Tarso I.E.D - Especialista en Educación y Gestión Ambiental - Magister en Educación de la Universidad de La Sabana. Correo: yolandaol@unisabana.edu.co

2 Universidad de La Sabana. Facultad de Educación. Maestría en Pedagogía. Correo: carlos.barreto2@unisabana.edu.co
} 
Bio - grafía. Escritos sobre la Biología y su Enseñanza. ISSN 2027-1034

Edición Extraordinaria. p.p. 1221 - 1229

Memorias del IX Encuentro Nacional de Experiencias en Enseñanza de la Biología y la

Educación Ambiental. IV Congreso Nacional de Investigación en Enseñanza de la

Biología.

scientific and technological competencies from the Teaching for Understanding $(\mathrm{EpC})$ approach based on the methodology of cooperative learning. It describes the established work path, the advantages and disadvantages of the methodology used, the learning process carried out, the acquired scientific competences, the use of technological tools and the interaction mechanisms for the training of citizens.

From the conceptual review and its contrast with the exercises performed in class, it was possible to establish the characteristics of the teaching practice with the students, generating processes of improvement and acceptance of the methodology of cooperative work that favor and optimize the learning in sciences Through the understanding of the contents and assertive socialization.

KEY WORDS: Teaching for understanding - Cooperative learning - Scientific competences - Citizen training - Use of TIC.

\section{INTRODUCCIÓN}

Esta investigación describe una estrategia pedagógica basada en el aprendizaje cooperativo cuyo propósito es identificar los alcances de esta metodología para la adquisición de competencias científicas desde el enfoque de la Enseñanza para la Comprensión como eje pedagógico del colegio Pablo de Tarso I.E.D. Se establece desde una planeación semiestructurada con unas estrategias de trabajo cooperativo en el aula de clase.

El Colegio Pablo de Tarso I.E.D tiene como principio institucional en su quehacer como institución pública la inclusión para sordos, la formación integral desde la perspectiva de la EpC mediante ambientes propicios para el desarrollo de competencias que potencien su proyecto de vida; de esta forma se contribuye con la conservación, protección del ambiente, el mejoramiento y la transformación de la sociedad.

Desde esta función pedagógica el trabajo en el aula busca desde el campo de la Ciencia y la Tecnología cumplir con dicha tarea a partir de metodologías incluyentes, dinámicas y de innovación pedagógica; que inicialmente fueron utilizadas para el trabajo en aulas virtuales, el uso de la informática educativa y las nuevas herramientas tecnológicas en el aula de clase para el trabajo en equipo y el aprender del otro y/o de otros, se convierte en una metodología donde el maestro es mediador en el proceso de aprendizaje, útil para la enseñanza del saber hacer en cualquier contexto; que como lo indica (Johnson,D; Johnson, E \& 


\section{Bio - grafía. Escritos sobre la Biología y su Enseñanza. ISSN 2027-1034}

\section{Edición Extraordinaria. p.p. 1221 - 1229}

Memorias del IX Encuentro Nacional de Experiencias en Enseñanza de la Biología y la Educación Ambiental. IV Congreso Nacional de Investigación en Enseñanza de la Biología.

Holubec,E., 1.999) permite optimizar el aprendizaje de todos los estudiantes, favorecen las relaciones entre estudiantes y contribuyen a la valoración de la diversidad en el aula de clase.

La metodología del Aprendizaje Cooperativo como estrategia pedagógica tiene como propósito promover el trabajo en equipo en los estudiantes, para lograr un objetivo común que beneficie a todos los miembros, potencie sus habilidades y permita un aprendizaje comprensivo de los temas estudiados. Así como lo refiere Deutsch (1993) al afirmar que a través del trabajo cooperativo se obtienen efectos positivos en términos de las relaciones sociales entre las personas, teniendo en cuenta sus diferencias en los niveles de habilidades, raza, género y clase social.

Desde el enfoque de la EpC el aula de clase es el lugar que favorece la comprensión de los procesos de investigación escolar y permite desarrollar las metas de comprensión a partir de unos tópicos generadores que a través de un cronograma planificado desarrollan los hilos conductores establecidos para la realización del trabajo cooperativo.

La metodología de trabajo en el aula de clase tiene como ambición transformar los métodos de enseñanza que utilizan los maestros (de transmisor de conocimiento a generador y mediador de conocimiento) y las representaciones que poseen los estudiantes y padres de familia acerca de cómo aprender y adquirir conocimiento. Sin embargo, el análisis cualitativo busca relacionar y determinar el alcanza al utilizar la metodología de aprendizaje cooperativo y el enfoque de enseñanza para la comprensión en la adquisición de competencias científicas por parte de los estudiantes; como lo explica Parra (1998) la actividad educativa se convierte en objeto de observación y explicación y como objeto de conocimiento (teórico) permite la comprensión objetiva de la práctica educativa. Habría que decir también al respecto que los aprendizajes generados se convierten en una construcción progresiva y conjunta entre el maestro y el estudiante como lo sustenta Jhonson,D \& Jhonson, R (1999).

\section{Contexto}

Este trabajo se desarrolló en el Colegio Pablo de Tarso I.E.D (Institución educativa distrital), ubicada en el barrio San Pablo segundo sector de la localidad de Bosa en la ciudad de Bogotá, D.C. La población de estudiantes de grado séptimo de educación básica secundaria es de 132 estudiantes en la jornada de la mañana; la población objeto de estudio fueron los estudiantes de grado 701 y 702 cuya número de estudiantes corresponde a 88 alumnos. El $95 \%$ de la población de estudiantes provienen de barrios de la localidad en estrato 1 y 2 ; con un rango de edad entre los 12 y 16 años. La población estudiantil atendida en la institución educativa en la jornada mañana es de 1530 estudiantes. 
Bio - grafía. Escritos sobre la Biología y su Enseñanza. ISSN 2027-1034

Edición Extraordinaria. p.p. 1221 - 1229

Memorias del IX Encuentro Nacional de Experiencias en Enseñanza de la Biología y la

Educación Ambiental. IV Congreso Nacional de Investigación en Enseñanza de la

Biología.

\section{METODOLOGÍA}

Al considerar la relación entre el alcance de la metodología de Aprendizaje Cooperativo y el enfoque de $\mathrm{EpC}$ en la adquisición comprensiva de competencias científicas en los estudiantes, el trabajo se enmarca en el enfoque cualitativo, busca comprender los acontecimientos que ocurren en el aula. Es descriptiva y desde el diseño de investigación, la Investigación Acción Pedagógica, Schôn, (1992) explica que es a través de la reflexión en la acción que el maestro construye saber pedagógico, de esta forma puede criticar su práctica pedagógica y transformarla; así la investigación acción pedagógica permite la deconstrucción de los procesos planteados en su práctica en el aula de clase, pero también proporciona la reconstrucción de dicha práctica pedagógica.

Teniendo en cuenta lo anterior se realiza una planeación semiestructurada del trabajo en el aula de clase, realizado con los estudiantes de grado séptimo, mediante las siguientes fases del proceso:

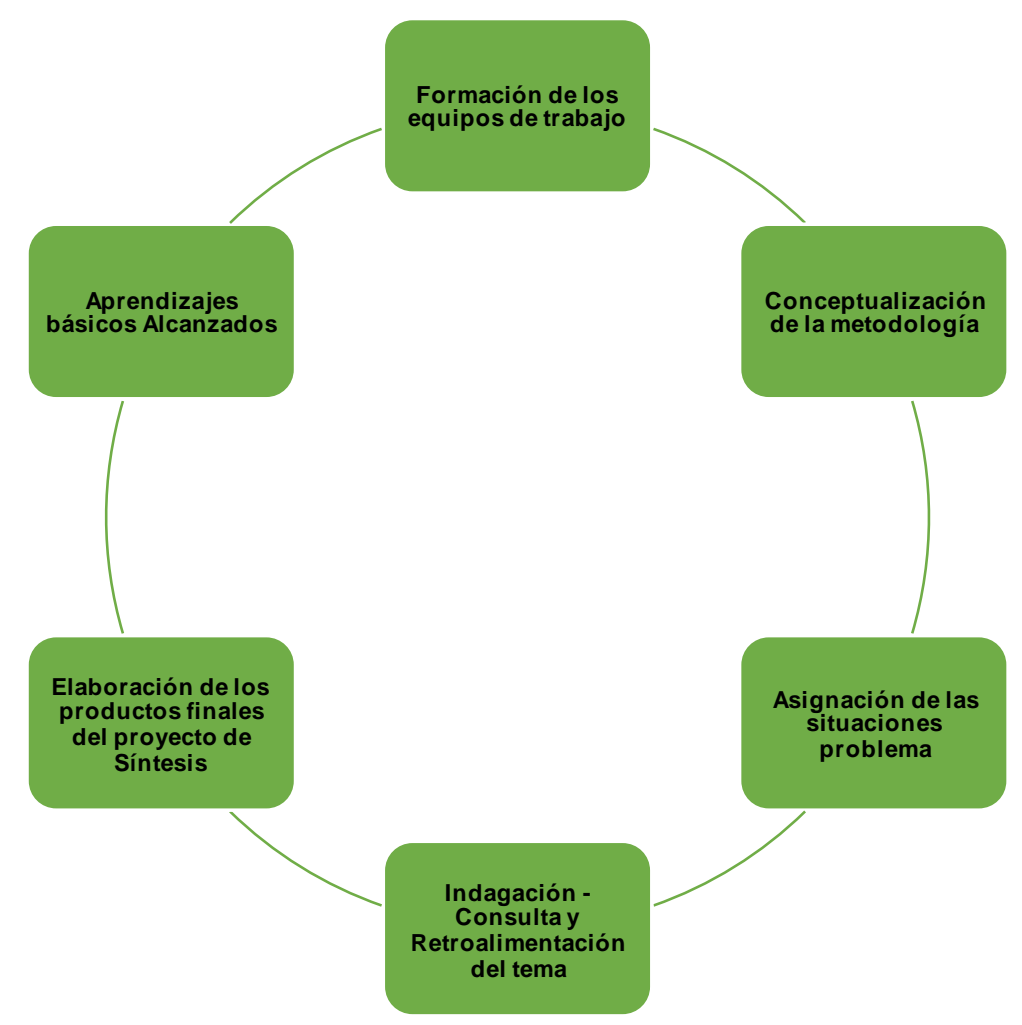

Figura No. 1 Fases del proceso establecidas en el proyecto de Aula 
Bio - grafía. Escritos sobre la Biología y su Enseñanza. ISSN 2027-1034

Edición Extraordinaria. p.p. 1221 - 1229

Memorias del IX Encuentro Nacional de Experiencias en Enseñanza de la Biología y la

Educación Ambiental. IV Congreso Nacional de Investigación en Enseñanza de la

Biología.

Teniendo en cuenta las fases del proceso en la aplicación de la metodología planteada y posterior a los resultados obtenidos en el trabajo realizado con los estudiantes se analizaron las siguientes categorías: Proceso de aprendizaje realizado por los estudiantes, competencias científicas adquiridas durante el proceso, manejo y uso de las herramientas tecnológicas, competencias intrapersonales e interpersonales en la formación de ciudadanos y por último alcance de la metodología planteada.

Con el propósito de recoger información de las categorías de análisis se diseñó un diario de clase donde el profesor describía los hechos observados para cada categoría analizada, realiza una interpretación de dichos hechos, realiza una comparación entre la teoría y la práctica y finalmente se obtiene el análisis y las conclusiones de la categoría estudiada.

\section{RESULTADOS Y DISCUSIÓN}

A continuación, se presentan los resultados obtenidos a partir del análisis de las fichas de diario de campo para cada categoría:

\section{Proceso de Aprendizaje Realizado por los Estudiantes}

De los 88 estudiantes del grado séptimo, 60 estudiantes mostraron un avance significativo en su proceso de aprendizaje; los estudiantes afirman que por el apoyo obtenido de los compañeros el trabajo era más fácil de realizar y comprendían mejor la información del tema trabajado, además que las tareas asignadas facilitaban la comprensión progresiva de los aprendizajes; sin embargo cuando por cualquier razón, generalmente de tiempos no estudiaban el desarrollo de las preguntas en el aula de clase, era mucho más dificil entender las temáticas. Conviene aclarar que las habilidades en la comprensión lectora de cada uno de los miembros del equipo facilitaban el aprendizaje del tema tratado al igual que el contar con competencias comunicativas como el saber escucharse, la asertividad, la expresión y sustentación de sus puntos de vista para la ejecución de las tareas planteadas en la interacción con el equipo de trabajo.

Los 28 estudiantes que no lograron mejorar sus procesos de aprendizaje mostraron dificultades en sus habilidades comunicativas en particular en la lectura comprensiva, dificultad para comprender y analizar información textual; pero además presentaron dificultad para desarrollar sus competencias interespecíficas como el manejo de conflictos, la capacidad para crear e imaginar figuras o textos, para darse a entender y plantear acuerdos con sus compañeros.

\section{Competencias Científicas Adquiridas en el Proceso}


Bio - grafía. Escritos sobre la Biología y su Enseñanza. ISSN 2027-1034

Edición Extraordinaria. p.p. 1221 - 1229

Memorias del IX Encuentro Nacional de Experiencias en Enseñanza de la Biología y la

Educación Ambiental. IV Congreso Nacional de Investigación en Enseñanza de la Biología.

Durante el aprendizaje adquirido en el desarrollo del proyecto se pudo evidenciar la siguiente escala de valoración.

\begin{tabular}{|c|l|c|}
\hline $\begin{array}{c}\text { Escala de } \\
\text { Valoración }\end{array}$ & Competencia científica Adquirida & $\begin{array}{l}\text { No. de estudiantes } \\
\text { valorados }\end{array}$ \\
\hline Alto & $\begin{array}{l}\text { Comprensión reflexiva y crítica de la } \\
\text { realidad planteada. } \\
\text { Dialogo asertivo con ideas } \\
\text { argumentadas. }\end{array}$ & \\
\hline Medio & $\begin{array}{l}\text { Relaciona conceptos disciplinares } \\
\text { con el desarrollo de avances } \\
\text { científicos y su aplicación } \\
\text { tecnológica. } \\
\text { Explica los hilos conductores. }\end{array}$ & \\
\hline Bajo & $\begin{array}{l}\text { Interpretación y contextualización } \\
\text { de fuentes de información } \\
\text { inadecuadas. 28 Estudiantes } \\
\text { Dificultad para el análisis crítico de } \\
\text { fuentes de información } \\
\text { las funsultadas. }\end{array}$ & \\
\hline
\end{tabular}

Cuadro No. 1 Competencias Científicas adquiridas en el Proyecto

Algunas afirmaciones del grupo al exponer su tema, dejan evidenciar que al adquirir competencias científicas se logran también mejorar las competencias comunicativas que perfeccionan los aprendizajes y la comprensión de los temas estudiados; Meinardi (2010) dice que la práctica de las ciencias es una actividad reflexiva donde las técnicas, destrezas y estrategias contribuyen a perfeccionar las competencias científicas.

\section{Manejo y Uso de las Herramientas Tecnológicas}

Hay que mencionar que quienes poseen los medios para realizar la búsqueda de información y cuentan con el seguimiento y apoyo de los padres y/o acudientes tienen mayor ventaja para obtener una información más precisa de los temas a trabajar.

De los 68 estudiantes, solo 18 estudiantes contaban con esta ventaja, los restantes realizaban una búsqueda incompleta, no acorde con la información 
Bio - grafía. Escritos sobre la Biología y su Enseñanza. ISSN 2027-1034

Edición Extraordinaria. p.p. 1221 - 1229

Memorias del IX Encuentro Nacional de Experiencias en Enseñanza de la Biología y la

Educación Ambiental. IV Congreso Nacional de Investigación en Enseñanza de la Biología.

requerida o se les dificultaba hacer uso de las herramientas de powerpoint y publisher. Lo que nos indica que aunque en apariencia conocen y manejan los medios tecnológicos si estos no son de uso continuo presentan deficiencias para su utilización. El grupo de 28 estudiantes no realizaron las tareas en el plazo acordada para su entrega, les faltó adquirir mayor grado de compromiso y responsabilidad, y además no contaban con la disponibilidad de estas herramientas tecnológicas en casa.

\section{Competencias Intrapersonales e Interpersonales}

Son también llamadas competencias emocionales que permiten identificar y responder constructivamente ante las emociones propias y las de los demás. En el trabajo en equipo aprender a leer al otro es una tarea fundamental, en esta edad el manejo de emociones como la rabia, la impotencia, la irritación, la disciplina de trabajo, la dependencia del otro, la falta de cooperación, los distractores de la edad, la falta de habilidades en algunos miembros del equipo; son factores que dificultan los procesos del aprendizaje cooperativo; sin embargo a través de la realización de las tareas planteadas se ven evocados a coexistir y generar acciones de apoyo, ayuda y motivación.

Teniendo en cuenta que estas consideraciones son inherentes a los seres humanos, un $10 \%$ presentó fricciones entre los miembros de los equipos de trabajo. Además, la falta de responsabilidad para la entrega de las tareas asignadas fue otra de las causas de inconvenientes no solo entre ellos mismos; se presentaron conflictos también entre los padres de familia y los docentes debido a no asumir sus compromisos como estudiante y al incumplimiento en el logro de los criterios de evaluación establecidos para la elaboración del proyecto.

\section{Alcance de la Metodología}

El aprendizaje cooperativo contribuye tanto en el mejoramiento del rendimiento académico de los estudiantes, como a la construcción de relaciones positivas entre ellos. Hay que mencionar, además que no siempre se logra este propósito, las condiciones sociales del medio en el que se forman los estudiantes (drogadicción, familias separadas, conflictos intrafamiliares) muchas veces son obstáculo para lograr dichos fines.

Por otro lado, la metodología de Aprendizaje Cooperativo permite apoyar y ayudar a los estudiantes que presentan dificultades en sus procesos de aprendizaje, los estudiantes que necesitan ayuda se sienten más tranquilos y en confianza.

Además, se demostró que los estudiantes que aprenden a partir de esta metodología son más competentes a nivel social y tienen mejores competencias ciudadanas (se vuelven más sociables, más extrovertidos, tienen mayor confianza en sí mismos). Para la ejecución del proyecto de síntesis se realizó un aprendizaje 
Bio - grafía. Escritos sobre la Biología y su Enseñanza. ISSN 2027-1034

Edición Extraordinaria. p.p. 1221 - 1229

Memorias del IX Encuentro Nacional de Experiencias en Enseñanza de la Biología y la

Educación Ambiental. IV Congreso Nacional de Investigación en Enseñanza de la

Biología.

cooperativo formal de esta manera se logró alcanzar las metas de comprensión y obtener las competencias específicas en el campo de la ciencia y la tecnología.

\section{CONCLUSIONES}

- La obtención de las características requeridas en los estudiantes para el aprendizaje cooperativo contribuye en mejorar las competencias científicas, si estas son enseñadas en edades tempranas.

- El enfoque de la enseñanza para la comprensión puede ser emprendido desde la metodología de aprendizaje cooperativo, puesto que contribuye a mejorar las comprensiones de los aprendizajes en el aula de clase.

- El aprendizaje cooperativo prepara para la convivencia social, en el logro de ese aprendizaje es importante involucrar la práctica de valores, lo que permite mejorar las acciones y los comportamientos como ciudadanos.

- La metodología de aprendizaje cooperativa es una manera de desarrollar además de la comprensión de los aprendizajes, la ejecución del enfoque de trabajo por proyectos.

- La distribución de los grupos debe potencializar y garantizar un balance tanto en las actividades académicas de los estudiantes como de sus habilidades y competencias sociales.

\section{REFERENCIAS}

- Deutsch, M., (1993). Educating for a peaceful world. American Psychologist, $48,510-517$.

- Gómez, P.M.\& Gómez H, A., (2016). Experiencia docente acerca del uso didáctico del aprendizaje cooperativo y el trabajo de campo en el estudio del fenómeno de influencia social. Revista electrónica interuniversitaria de 
Bio - grafía. Escritos sobre la Biología y su Enseñanza. ISSN 2027-1034

Edición Extraordinaria. p.p. 1221 - 1229

Memorias del IX Encuentro Nacional de Experiencias en Enseñanza de la Biología y la

Educación Ambiental. IV Congreso Nacional de Investigación en Enseñanza de la Biología.

formación de profesores. V 19 (2), 331-346. DOI: http://dx.doi.org/10.6018/reifop.19.2.206921

- Johnson, D., Johnson, E \& Holubec, E. (1.999). El aprendizaje cooperativo en el aula. Buenos Aires. Paidós.

- Meinardi, E. (2010). Educar en Ciencias. Voces de la Educación.

- Parra, C., (1998). Naturaleza de la acción educativa. Educación y Educadores. Universidad de la Sabana. 25-40.

- Schön, D. (1992). La formación de profesionales reflexivos. Hacia un nuevo diseño de la enseñanza y el aprendizaje en las profesiones. Editorial Paidós, Barcelona. 